\title{
Equivalent modulus method for finite element simulation of the sound absorption of anechoic coating backed with orthogonally rib-stiffened plate
}

\author{
Zhongkun Jin, Yao Yin, Bilong Liu* \\ Key Laboratory of Noise and Vibration Research, Institute of Acoustics, \\ Chinese Academy of Sciences, 100190, Beijing, P.R.China
}

\begin{abstract}
The finite element method is often used to investigate the sound absorption of anechoic coating backed with orthogonally rib-stiffened plate. Since the anechoic coating contains cavities, the number of grid nodes of a periodic unit cell is usually large. An equivalent modulus method is proposed to reduce the large amount of nodes by calculating an equivalent homogeneous layer. Applications of this method in several models show that the method can well predict the sound absorption coefficient of such structure in a wide frequency range. Based on the simulation results, the sound absorption performance of such structure and the influences of different backings on the first absorption peak are also discussed.

Keywords: finite element method; homogenization; sound absorption; anechoic coating; orthogonally rib-stiffened; equivalent modulus.
\end{abstract}

\section{Introduction}

The Alberich anechoic coating with embedded air-filled cavities is often used in underwater vehicles to absorb sound energy. Two absorption peaks always exist in the low-frequency band when periodically distributed cavities are contained in the anechoic coating. The acoustic resonances of the cavities result in strong vibration of the coating; for transverse waves rapidly dissipated in viscoelastic media, a vigorous redistribution of sound waves in the lateral directions of the cavities causes substantial energy dissipation in the vicinity of the resonance frequencies [1]. Therefore, the

* Corresponding author. Tel: +86 1082547538

E-mail addresses: liubl@mail.ioa.ac.cn. 
coating exhibits significant sound absorption at the acoustic resonances of the cavities.

The finite element method (FEM) combined with the Bloch theorem has been developed to analyze the sound absorption performance of singly periodic structures, such as compliant gratings [2,3], and also doubly periodic structures, such as the Alberich anechoic coatings $[4,5]$. It is widely used to predict the sound absorption performance of periodic structure with different shapes. To compute the acoustic performance, one periodical unit cell of the structure needs to be meshed using the Bloch theorem. The criterion of mesh size was discussed in the literature [4] which pointed out that if the transverse wave velocity and longitudinal wave velocity were relevant parameters, the $\lambda / 4$ criterion with respect to transverse wave would be sufficient; otherwise, the $\lambda / 4$ criterion with respect to flexural wave would be needed when the flexural wave velocity was relevant.

Furthermore, to reveal the absorption mechanism of the Alberich anechoic coating, the influences of locally resonant modes on sound absorption were investigated [6]. In recent studies, the acoustic performance of anechoic coating backed with steel plates was discussed, and the coating and backing were treated as a whole structure [1]. Then, the optimization scheme for the physical and structural parameters was discussed, and it was verified by experiments that combinations of different locally resonant units could provide significant sound absorption at low frequencies over a broad band when the optimized parameters were used [7].

In engineering practice, the anechoic coating adheres to shells internally and orthogonally stiffened by sets of stringers and frames, such as ship hulls. Usually, the distance between the stringers or frames is larger than the distance between the cavities embedded in the coating. Thus, the periodical unit cell of the hull contains an orthogonal stiffener and lots of cavities. To precisely capture the geometrical features of cavities in the FEM, a large number of grid nodes is required if the unit cell is directly meshed, making the numerical operation difficult.

The acoustic performance of rib-stiffened homogeneous plates has been studied with analytical methods $[8,9]$, but without plate with embedded cavities. Recently, a 
2-D FEM was used to investigate the vibro-acoustic response of an infinite rib-stiffened thick-plate assembly that contains infinitely long cavities [10]; however, the actual features of cavities with finite sizes could not be modeled. The sound absorption performance of a rib-stiffened plate covered by the Alberich anechoic coating has just been investigated using a simplified FEM [11], in which the ribs were arranged along only one direction and the shifting of the main absorption peak was treated by the impedance transfer approach. This simplified method can significantly reduce the calculation time, but the calculation is limited in a narrow frequency band containing only one main absorption peak.

In this paper, an equivalent modulus method also based on the impedance transfer formula is proposed for the homogenization of the finite element model simulating the absorption performance of structures with large-size unit cells over a broader frequency band. With this method, the number of grid nodes of the finite element meshes is greatly reduced, and the low-frequency response of an Alberich anechoic coating backed by orthogonally rib-stiffened plate can be predicted more accurately in a wider frequency range. The paper is organized as follows: Section 2 describes a finite element model constructed to simulate the acoustic performance of an infinite anechoic coating backed with steel plate periodically stiffened by orthogonal sets of ribs, and verification of the numerical algorithm; the proposed equivalent modulus method with two schemes and the applications of the two schemes in the same finite element model is introduced in Section 3; Section 4 presents further applications and discussions on the second equivalent modulus scheme; based on the simulation results of several models, some patters of variation of the first absorption peak of Alberich anechoic coating backed by orthogonally rib-stiffened plates are discussed in Section 5; finally, the conclusion of this research is presented in Section 6.

\section{Finite element model}

In this section, a finite element model is constructed to simulate the acoustic performance of an infinite anechoic coating backed with a plate periodically stiffened 
by orthogonal ribs. The numerical algorithm is then verified by solving a problem with known solution.

\subsection{Finite element modeling and the Bloch theorem}

The physical problem under consideration is the incidence of a pressure wave traveling in the $z$-direction on the bottom surface of a structure composed of the Alberich anechoic coating and an orthogonally rib-stiffened steel plate, as shown in Fig. 1. The anechoic coating that contains air cavities (cylinder-shaped) is made up of viscoelastic rubber material. The steel plate is bonded to the coating and orthogonal ribs are affixed to the steel plate. Such a structure is assumed to be infinite in the $x$ and $y$ directions. In this study, the fluid loaded on the side of anechoic coating is semi-infinite water, and that above the steel plate and ribs is the air, which is neglected in the finite element analysis. The cavities are periodically arranged in both the $x$ and $y$ directions, and the ribs attached to the steel plate are also equally spaced in the $x$ and $y$ directions, so both the cavities and ribs are periodically distributed.

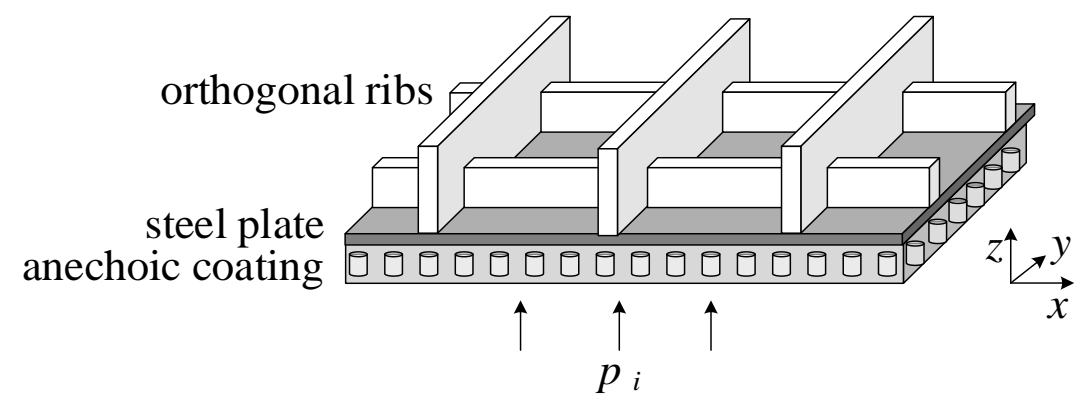

Fig. 1. Sketch of an Alberich anechoic coating backed with orthogonally rib-stiffened plate. A plane wave is incident from the semi-infinite water domain.

In the FEM model of such a structure, the Bloch theorem for periodic structures is applied, so only one unit cell of the structure needs to be meshed. Figure 2(a) shows a sketch of the unit cell, which consists of a set of orthogonal ribs, a homogeneous steel plate and an anechoic coating layer with embedded cavities. A part of the fluid 
domain below the anechoic coating is also included in the unit cell, but this is not shown in the figure.

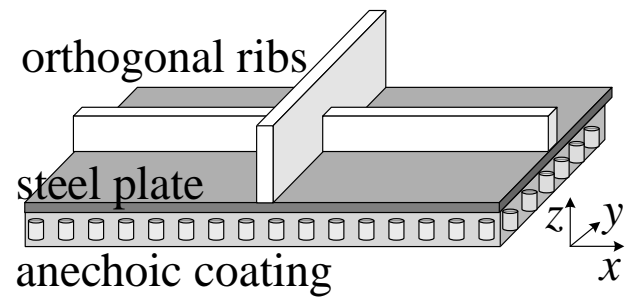

(a)

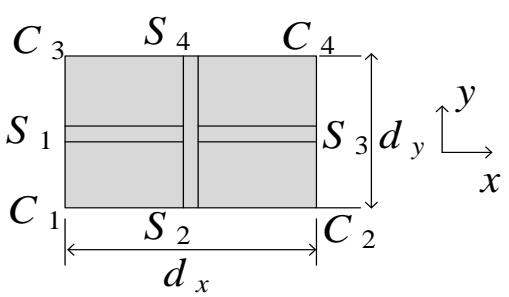

(b)

Fig. 2(a) Calculating model of a unit cell. (b) Top view of a unit cell, the description of the borders, including the $C_{1}, C_{2}, C_{3}, C_{4}$ lines and $S_{1}, S_{2}, S_{3}, S_{4}$ surfaces.

For periodic structure in the $x$ and $y$ directions, any space function $\chi$, such as the pressure and displacement, satisfies the equation

$$
\chi\left(x+d_{x}, y+d_{y}, z\right)=\chi(x, y, z) e^{\mathrm{j} k d_{x} \sin \theta \cos \varphi} e^{\mathrm{j} k d_{y} \sin \theta \sin \varphi}
$$

where $k$ is the wave number; $d_{x}$ and $d_{y}$ denote the periodic distances in the $x$ and $y$ directions, respectively; $\mathrm{j}$ is the complex unit; and $\theta$ and $\varphi$ are the direction angles of the incident wave $(\theta$ is the angle between the incident wave vector and the positive $z$-axis, and $\varphi$ is the angle between the projection in the $x y$-plane of the incident wave vector and the positive $x$-axis). In this research, the incident wave is parallel to the $z$-axis, so $\theta$ is zero.

An important application of the periodical boundary conditions (Eq. (1)) is the condensation of degrees of freedom of the unknown pressures and displacements. As shown in Fig. 2(b), the unit cell is divided into nine parts, including four corner lines $\left(C_{1}, C_{2}, C_{3}\right.$, and $\left.C_{4}\right)$, four surfaces $\left(S_{1}, S_{2}, S_{3}\right.$, and $\left.S_{4}\right)$ and the inner volume domain. The four lines and four surfaces are all perpendicular to the $x y$-plane. The distances between $C_{1}$ and $C_{2}, C_{3}$ and $C_{4}, S_{1}$ and $S_{3}$ are equal to $d_{x}$, while those between $C_{1}$ and $C_{3}, C_{2}$ and $C_{4}, S_{2}$ and $S_{4}$ are equal to $d_{y}$. Thus, the fluid pressure and structure displacement are split into nine corresponding parts, and according to Eq. (1), the space functions $\chi$ of the nine parts satisfy the following relations 


$$
\begin{aligned}
& \chi_{S_{3}}=\chi_{S_{1}} e^{\mathrm{j} \psi_{x}}, \quad \chi_{S_{4}}=\chi_{S_{2}} e^{\mathrm{j} \psi_{y}} ; \\
& \chi_{C_{2}}=\chi_{C_{1}} e^{\mathrm{j} \psi_{x}}, \quad \chi_{C_{3}}=\chi_{C_{1}} e^{\mathrm{j} \psi_{y}}, \quad \chi_{C_{4}}=\chi_{C_{1}} e^{\mathrm{j}\left(\psi_{x}+\psi_{y}\right)}
\end{aligned}
$$

where $\psi_{x}=k d_{x} \sin \theta \cos \varphi$ and $\psi_{y}=k d_{y} \sin \theta \sin \varphi$.

The finite element matrix equation of fluid-structure coupling problem can be found in reference [4]. Substituting the above relations into the matrix equation, the condensation of degrees of freedom belonging to $S_{3}, S_{4}, C_{2}, C_{3}$ and $C_{4}$ are operated. The fluid pressure and structure displacement are obtained by solving the matrix equation; subsequently, the reflection coefficient $R$ and transmission coefficient $T$ are obtained. Finally, the absorption coefficient $\alpha$ is calculated from the following relation

$$
\alpha=1-|R|^{2}-|T|^{2}
$$

In this study, air termination is assumed in all the calculation models, so the transmission coefficient $T=0$.

\subsection{Verification of the numerical algorithm}

A numerical algorithm based on the finite element model introduced above is constructed in MATLAB. In this section, the accuracy of the model and algorithm is verified by simulating a numerically solved problem. The upper frequency bound in the numerical analysis is set to be $6000 \mathrm{~Hz}$.

According to the modal expansion of reflection wave in the literature [4], below the cut-off frequency, plane wave parallel to the $z$-axis propagates away from the structure and the waves of other modes are all evanescent. Hence the sound pressure distribution in a unit cell is simulated. Referring to the structure shown in Fig. 2, the anechoic coating is assumed to be $40 \mathrm{~mm}$ thick with a grating spacing of $20 \mathrm{~mm}$. The thickness of the steel plate is $10 \mathrm{~mm}$, and the ribs are $6 \mathrm{~mm}$ thick. Considering that the space between stringers and the height of stringers are both different from those of frames, the length and height of the rib parallel to the $z y$-plane are set to be $120 \mathrm{~mm}$ and $20 \mathrm{~mm}$, respectively, and those of the rib parallel to the $z x$-plane are set to be 40 
$\mathrm{mm}$ and $10 \mathrm{~mm}$, respectively. The anechoic coating contains 12 square gratings in the unit cell, with each grating containing a cylindrical cavity with $30 \mathrm{~mm}$ height and 12 mm diameter. The physical parameters are assumed not to vary with frequency and they are selected as follows: for the viscoelastic material, the Young's modulus $E=4.5 \times 10^{7} \mathrm{~Pa}$; density $\rho=1100 \mathrm{~kg} / \mathrm{m}^{3}$; Poisson's ratio $\nu=0.49$; and loss factor $\mu=0.23$. For the steel plate and ribs, the Young's modulus is $2.1 \times 10^{11} \mathrm{~Pa}$; density is $7890 \mathrm{~kg} / \mathrm{m}^{3}$; and the Poisson's ratio is 0.3 . A $50 \mathrm{~mm}$ thick layer of water domain lies below the anechoic coating; the density of water is $1000 \mathrm{~kg} / \mathrm{m}^{3}$, and the sound speed is 1489 $\mathrm{m} / \mathrm{s}$.

The incident plane wave is perpendicular to the $x y$-plane, and the first cut-off frequency of the described model is $12400 \mathrm{~Hz}$, so the upper bound frequency (6000 $\mathrm{Hz}$ ) in numerical analysis is below the cut-off frequency and both the reflection wave and total sound pressure are also plane wave perpendicular to the $x y$-plane.

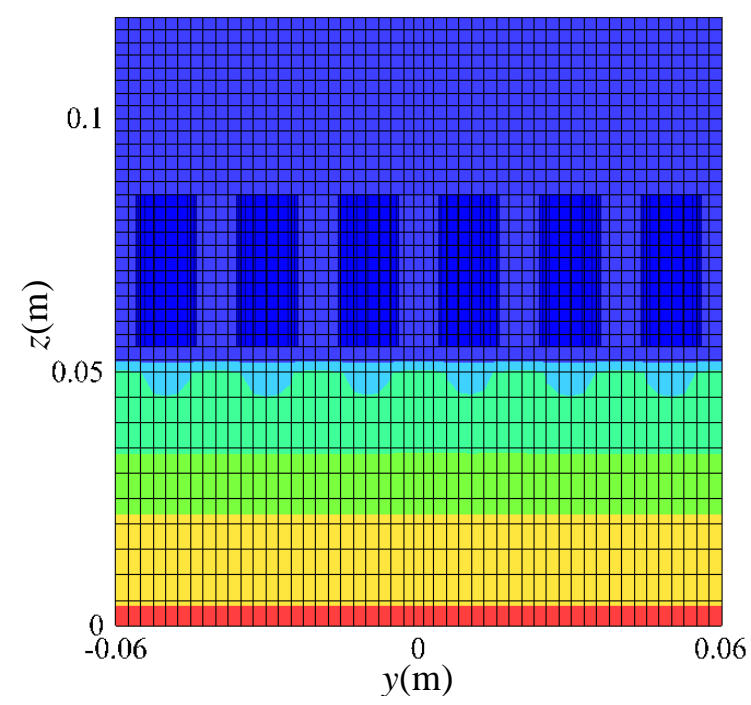

(a)

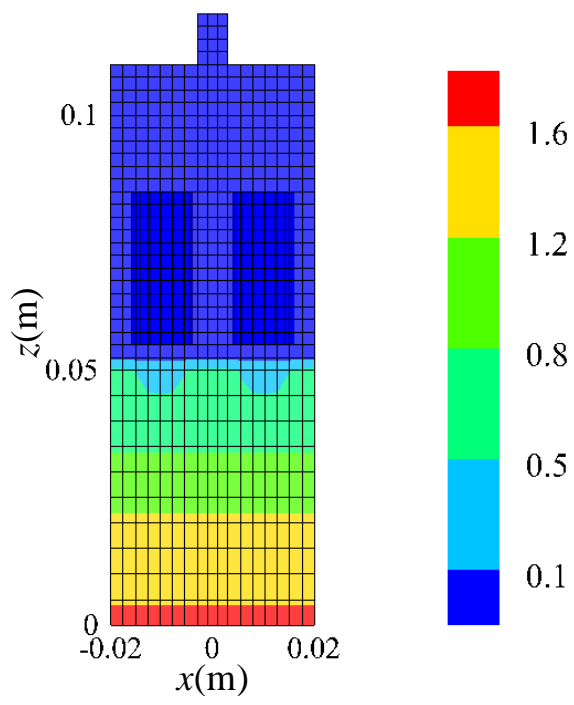

(b)

Fig. 3. Sound pressure field of the unit cell of an Alberich anechoic coating backed with orthogonally rib-stiffened plate at $6 \mathrm{kHz}$ : (a) in the $z y$-plane; (b) in the $z x$-plane.

The calculated sound field in the unit cell is shown in Fig. 3. The grids observing the $\lambda / 4$ criterion determined by the wavelength of flexural wave is also displayed. The sound pressure in the solid domain $(z>0.05 \mathrm{~m})$ is zero. Fig. 3(a) exhibits the sound 
field in a cross section parallel to the $z y$-plane, and Fig. 3(b) shows that parallel to the $z x$-plane. Sound waves other than the 00 mode also exist in the vicinity of the interface between the structure and fluid near the location of $0.05 \mathrm{~m}$ on the $z$-axis. However, they vanish rapidly with the increase of distance away from the interface. Hence, in the region near $0 \mathrm{~m}$ on the $z$-axis, only plane wave is observed.

\section{The equivalent modulus method}

If the lengths of ribs are much larger than the grating spacing of the Alberich anechoic coating, lots of cavities will be contained in the unit cell. In the finite element model of such a structure, the number of grid nodes must be very large and numerical operation would be difficult if the unit cell is directly meshed.

Hence, the homogenization procedure has been employed to replace the inhomogeneous material with a layer of homogeneous material, whose mechanical behavior remains to be same as the inhomogeneous material; in this way, a large number of grids are saved. Several homogenization methods have been proposed in previous studies [12-16] adapted to different physical problems. To calculate the effective sound velocity in an inhomogeneous mixture of two-phase materials, some complex empirical formulas were established based on the volume concentration of each component $[12,13]$. Based on experimental or numerical data, some theoretical approaches were developed to compute the equivalent elastic modulus of honeycomb $[14,15]$. The equivalent complex moduli of laminated glass beams were also obtained with a purely experimental method [16].

However, these homogenization methods are not suitable for the model under consideration because in the physical problem, the resonant absorption effect is affected by the backing conditions.

In this section, an equivalent modulus method is proposed for the homogenization procedure in the current model, and two equivalent schemes based on the impedance transfer formula are developed. The first scheme has two main steps: 1) calculate the reflection coefficient $R$ of an Alberich anechoic coating with air backing and the 
absorption coefficient using Eq. (3), and then the acoustic impedance $Z$ at the reflection interface $(Z=(1+R) /(1-R))$. Next, calculate the complex equivalent sound velocity based on the acoustic impedance in the impedance transfer formula, and then the equivalent moduli of a homogeneous anechoic coating is obtained. 2) Calculate the absorption coefficient of the equivalent homogeneous coating backed with an orthogonally rib-stiffened plate representing the original inhomogeneous structure. The second scheme begins with calculating the absorption coefficient of an Alberich anechoic coating backed with steel plate, and the rest of the procedure is similar to the first scheme.

\subsection{The first equivalent modulus scheme}

The absorption coefficients of an Alberich anechoic coating with air backing are first calculated with the FEM model. The parameters of the anechoic coating are identical with the verification problem in Section 2.2. According to the FEM model and Bloch theorem introduced above the calculating unit cell contains only one grating, likewise one cavity. The curve of absorption coefficient versus frequency is demonstrated in Fig. 4, and two peaks are found around $2150 \mathrm{~Hz}$ and $6600 \mathrm{~Hz}$. At the peak frequencies, substantial mode conversion from longitudinal wave to transverse wave around the cavity could enhance the dissipation of acoustic energy [6].

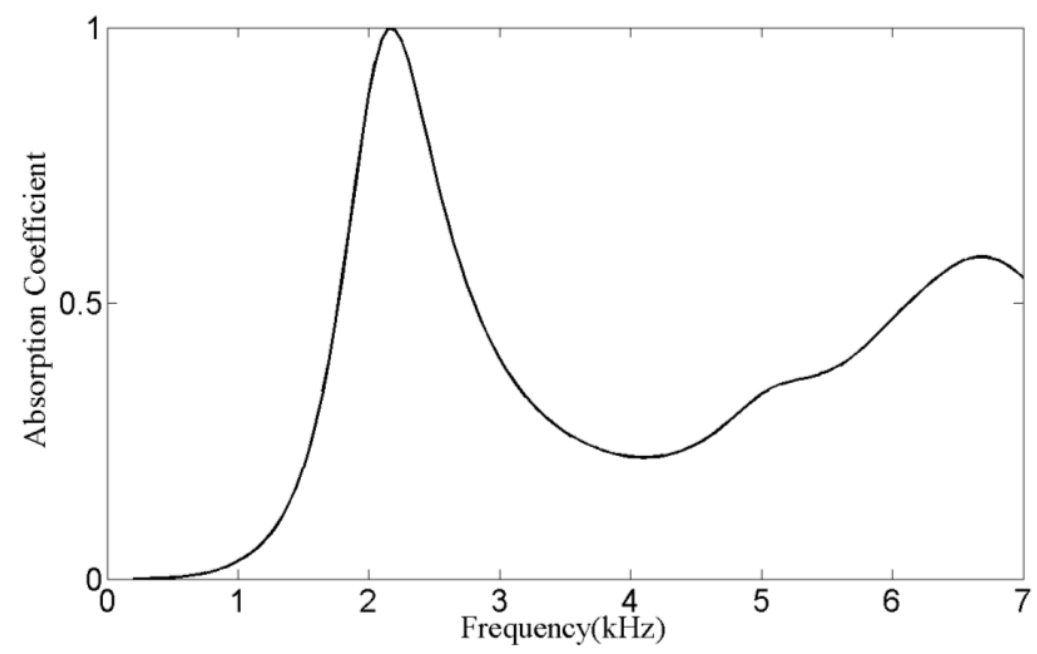

Fig. 4. Absorption coefficients of an Alberich anechoic coating with air backing. 
Now consider a homogeneous layer with air backing whose thickness and input impedance are equal to those of the Alberich anechoic coating. According to the transfer impedance formula [17]

$$
Z_{i}=\rho c \frac{Z_{l}+\mathrm{j} \rho c \tan k l}{\rho c+\mathrm{j} Z_{l} \tan k l}
$$

where $\rho, c, k$ and $Z_{i}$ represent the density, sound velocity, wave number and input impedance of the layer, respectively, and $Z_{l}$ is the impedance at the location with distance $l$ away from the input surface. Hence the input impedance for a homogeneous layer with air termination $\left(Z_{l}=0\right)$ is

$$
Z_{i}=\mathrm{j} \rho c \tan (k l)
$$

We assume that $\rho$ is the mean density of Alberich anechoic coating, $866.7 \mathrm{~kg} / \mathrm{m}^{3}$. Using the Monte Carlo iterative method [18], the nonlinear Eq. (5) is solved to get the complex sound velocity $c$ of the equivalent homogeneous layer for each frequency. Then, the equivalent Young's modulus $E$ and loss factor $\mu$ for each frequency are derived through the relation

$$
c=\sqrt{\frac{E(1+\mathrm{j} \mu)(1-\sigma)}{(\rho(1+\sigma)(1-2 \sigma))}}
$$

In the calculation, the Poisson's ratio $\sigma$ is also assumed to be 0.49 .

The calculated equivalent Young's moduli and loss factors are exhibited versus frequency in Fig. 5(a) and Fig. 5(b), respectively. They both fluctuate with the frequency. Since the air-filled cavity is much softer than the viscoelastic material, the equivalent Young's moduli of the homogeneous coating are about one tenth of that of the original viscoelastic rubber material. The equivalent loss factors vary around the original loss factor. The absorption coefficients of the equivalent homogeneous layer with air backing calculated with the equivalent parameters are the same as those shown in Fig. 4.

The absorption coefficients of an Alberich anechoic coating and the corresponding equivalent homogeneous coating, backed with the same orthogonally rib-stiffened plate are then calculated, and the results are shown in Fig. 6, in which the solid curve and the dotted curve denote the results of the original model and the equivalent model, respectively. The former is called the original model, and the latter is called the equivalent model in the following discussions. Considering the computing capability in this study (an HP Workstation Z800, with $3.2 \mathrm{GHz}$ quad-core processer and 24G 
RAM), the lengths of the ribs are set to be a little larger than the grating spacing of the anechoic coating. The geometric and physical parameters of the original model are set to be the same as those of the verification model in Section 2.2, and the outer contour of the equivalent model composed of the ribs, steel plate and homogeneous anechoic coating are identical with the original model. The Young's moduli and loss factors of the equivalent model are shown in Fig. 5, and the other physical parameters are the same as the verification model in Section 2.2.

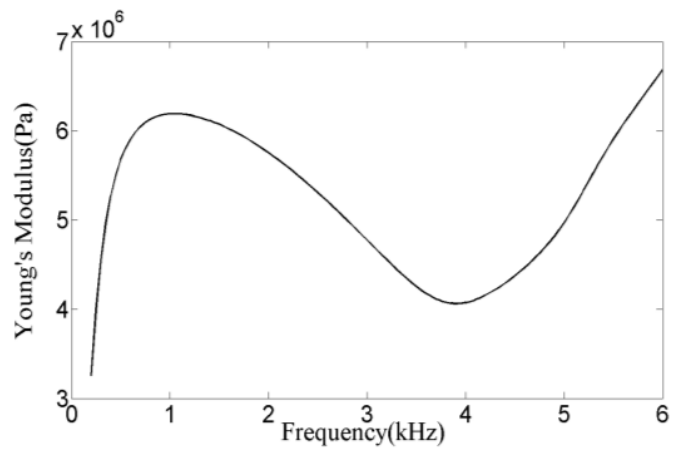

(a)

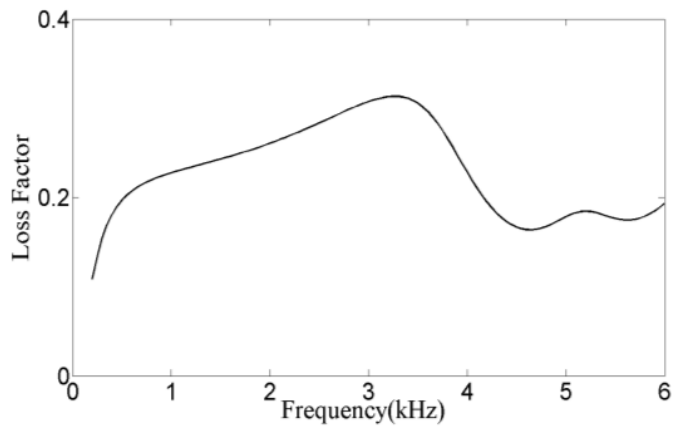

(b)

Fig. 5. Equivalent Young's moduli and loss factors derived from an Alberich anechoic coating with air backing: (a) Young's moduli; (b) loss factors.

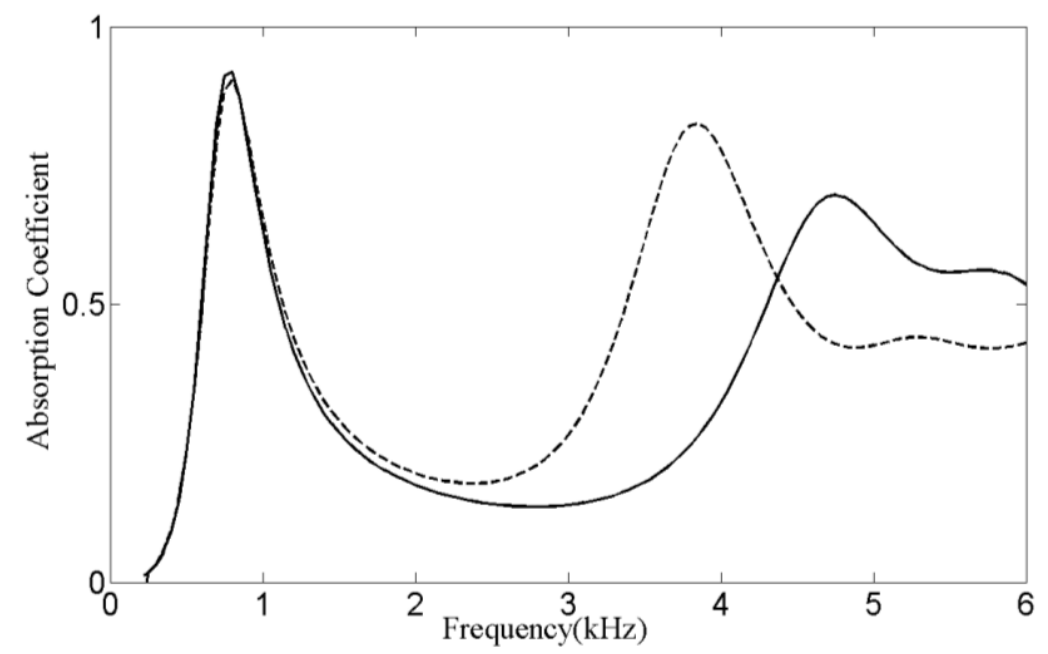

Fig. 6. Absorption coefficients of an Alberich anechoic coating and the equivalent homogeneous coating, backed with the same orthogonally rib-stiffened plate. The equivalent physical parameters required in the calculation are the same as those of the Alberich anechoic coating with air backing. Solid curve: Alberich anechoic coating. Dotted curve: equivalent homogeneous coating. 
Figure 6 shows that at frequencies below $1500 \mathrm{~Hz}$, except for a little difference at the peak frequency, the absorption coefficients of the two models agree well with each other; however, the results significantly diverge at higher frequencies. The first absorption peak is around $800 \mathrm{~Hz}$ both in the results of the original model and the equivalent model; however, the second absorption peak in the original model is around $4750 \mathrm{~Hz}$, while that of the equivalent model is around $3850 \mathrm{~Hz}$. This discrepancy is very likely caused by the scattering inside the Alberich anechoic coating of sound with shorter wave length in the higher frequency band. It further suggests that the equivalent moduli derived from an Alberich anechoic coating with air backing are no longer suitable for the same coating backed with orthogonally rib-stiffened steel plate.

It is worth mentioning that the simplified FEM proposed in reference [11] is very similar to this equivalent modulus scheme; for the same reason, that method is also only applicable in a narrow low-frequency band.

\subsection{The second equivalent modulus scheme}

In light of the analysis above, when air cavities are embedded in the anechoic coating, the acoustic scattering inside the coating will be affected by the conditions of backing, hence the equivalent modulus. Therefore, the second equivalent modulus scheme is proposed, which starts the homogenization procedure from an Alberich anechoic coating backed with steel plate.

Firstly, the absorption coefficient of such a structure is calculated, and the result is shown in the solid curve with two peaks at around $800 \mathrm{~Hz}$ and $4750 \mathrm{~Hz}$ in Fig. 7. The parameters of the anechoic coating in the unit cell are identical with those in Section 3.1, and the calculating unit cell also contains only one grating with one embedded cavity. The same size of orthogonally rib-stiffened plate in Section 3.1 is applied, and the mean mass of the ribs loaded on one grating of the anechoic coating is added to the original steel plate, so the thickness of the steel plate in the unit cell is increased to $13.5 \mathrm{~mm}$. 


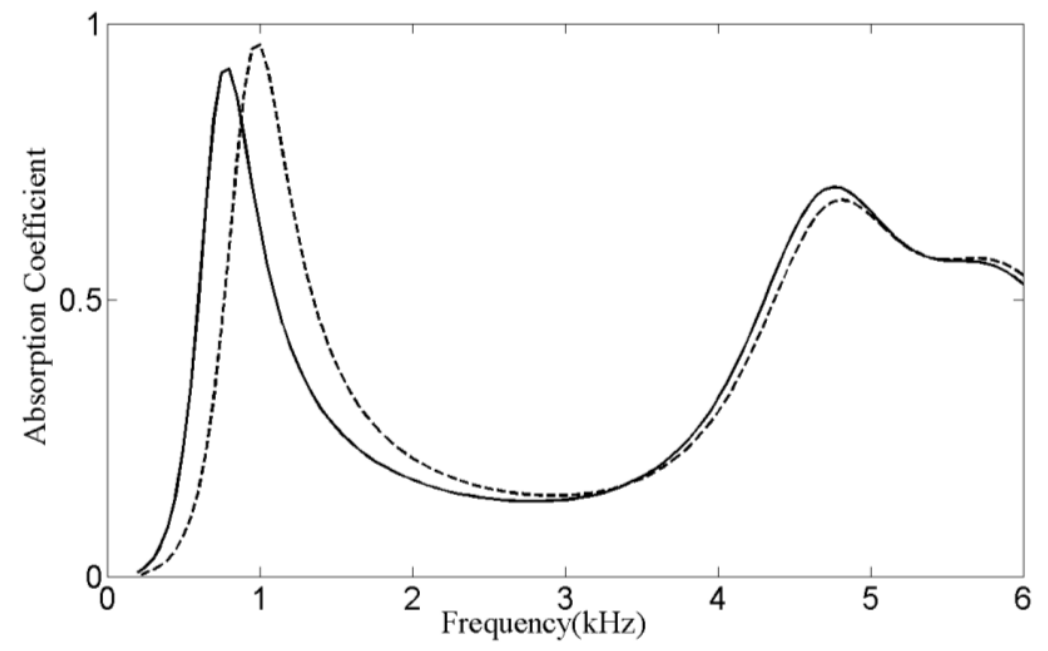

Fig. 7. Absorption coefficients of the Alberich anechoic coating backed with different steel plates.

Solid curve: plate with $13.5 \mathrm{~mm}$ thickness. Dotted curve: plate with $7.7 \mathrm{~mm}$ thickness.

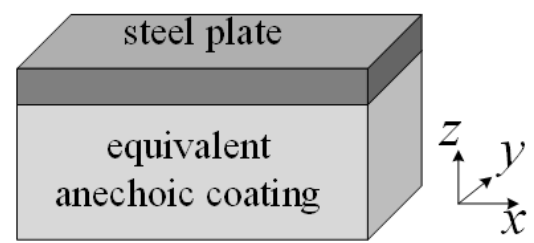

Fig. 8. Sketch of an equivalent homogeneous anechoic coating backed with steel plate.

Next, the transfer impedance formula (Eq. (4)) is applied twice to get the sound speed of the equivalent homogeneous coating backed with steel plate. For the model shown in Fig. 8, Eq. (4) is firstly used to derive the input impedance $Z_{l}$ at the interface between the steel plate and anechoic coating. In this step, because the steel plate is backed with air, we can use the simplified formula Eq. (5) to get the input impedance $Z_{l}=\mathrm{j} \rho_{\text {steel }} c_{\text {steel }} \tan \left(k_{\text {steel }} l_{\text {steel }}\right)$, where the subscript 'steel' represents the related item of the steel plate and $c_{\text {steel }}$ is calculated with Eq. (6) by using steel's parameters. Secondly, Eq. (4) is applied again to calculate the complex sound velocity $c$ of the equivalent homogeneous layer for each frequency by using the method introduced in Section 3.1. In this calculation, the impedance $Z_{l}$ obtained by the steel plate, $Z_{i}$ obtained by the Alberich anechoic coating backed with steel plate, the mean density $\rho$ and thickness $l$, are all known quantities.

Then, the equivalent Young's moduli and loss factors are obtained by solving Eq. (6) and the results are shown in solid curves in Fig. 9(a) and Fig. 9(b), respectively. 
Although the equivalent Young's moduli are also about an order of magnitude smaller than the original Young's modulus, the trend of variation is quite different from that shown in Fig. 5(a). Below $4000 \mathrm{~Hz}$, the equivalent loss factors vary smoothly around the original loss factor, but rise up sharply when the frequency exceeds $4000 \mathrm{~Hz}$.

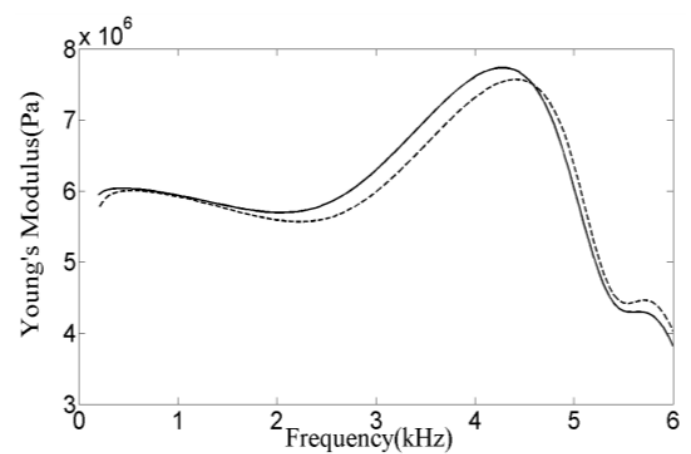

(a)

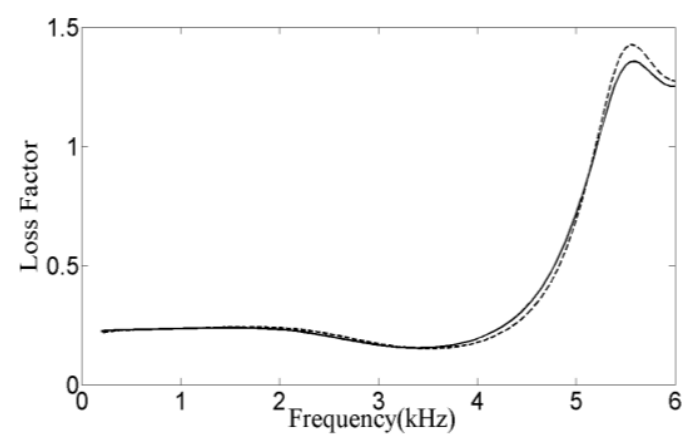

(b)

Fig. 9. Equivalent Young's moduli and loss factors derived from the same Alberich anechoic coating backed with different steel plates: (a) Young's moduli; (b) loss factors. Solid curves: plate with $13.5 \mathrm{~mm}$ thickness. Dotted curves: plate with $7.7 \mathrm{~mm}$ thickness.

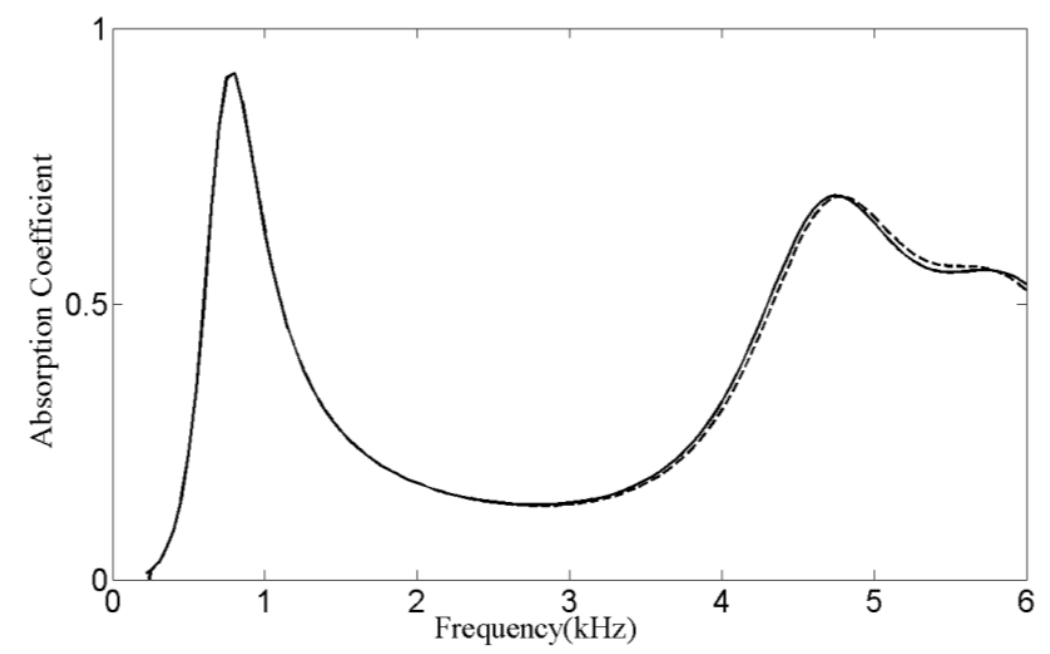

Fig. 10. Absorption coefficients of an Alberich anechoic coating and the equivalent homogeneous coating, backed with the same orthogonally rib-stiffened plate. The equivalent physical parameters required in the calculation are the same as those of the Alberich anechoic coating backed with steel plate. Solid curve: Alberich anechoic coating. Dotted curve: equivalent homogeneous coating.

The absorption coefficients of the Alberich anechoic coating and the corresponding equivalent homogeneous coating, both backed with the same orthogonally 
rib-stiffened plate are then calculated following the same procedure in Section 3.1, and the results are shown in Fig. 10 (the solid curve denotes the result of the original model and the dotted curve represents that of the equivalent model.). These two models are identical with the corresponding models described in Section 3.1, except for the physical parameters of the equivalent model. However, the results of the original and equivalent models are almost identical in the whole frequency band, both with two peaks at around $800 \mathrm{~Hz}$ and $4750 \mathrm{~Hz}$.

It is noted that the equivalent scheme can save a lot of computational memory and time. The number of the grid nodes of the original model is about $3.8 \times 10^{4}$ and the calculation time for 120 frequencies is about 130 hours, but the equivalent model only has $3.5 \times 10^{3}$ grid nodes and the calculation only takes 3 hours.

\section{Further applications and discussions of the second equivalent modulus scheme}

In this section, further applications of the second equivalent modulus scheme are introduced and discussed.

An additional application of the second equivalent modulus scheme is introduced in Section 4.1. This method is not only suitable for the model with voided cavities, but also for the slab model with local resonance, for example, caused by a hard core scatterer coated by a layer of soft silicon rubber. Another application of the second equivalent modulus scheme in model with large-size unit cell is introduced in Section 4.3 .

In Section 4.2, the vibration modes of the Alberich anechoic coating model are analyzed based on the displacement vectors at the resonant frequencies of the original model and equivalent model (similar results would also be obtained for the local resonance slab model). A model with evident shear deformation is also simulated and analyzed to illustrate a meshing rule under certain conditions and to further verify the equivalent modulus method.

The geometric parameters of the unit cells in all models introduced in Section 3 and 4 are also listed in Table 1. 
Table 1

Overall sizes of the unit cells in models.

\begin{tabular}{lllllll}
\hline Geometric parameters & \multicolumn{2}{l}{ Size $(\mathrm{mm})$} & & & \\
\cline { 2 - 6 } & Model 1 & Model 2 & Model 3 & Model 4 & Model 5 & Model 6 \\
\hline Thickness of coating layer & 40 & 40 & 40 & 40 & 40 & 40 \\
Grating spacing of coating layer & 20 & 20 & 20 & 20 & 20 & 20 \\
Thickness of steel plate & 10 & 10 & 10 & 4 & 10 & 6 \\
Thickness of ribs & 6 & 6 & 6 & 4 & 6 & 6 \\
Length/height of the rib parallel to $z x$-plane & $40 / 10$ & $40 / 10$ & $40 / 10$ & $40 / 100$ & $500 / 100$ & $500 / 100$ \\
Length/height of the rib parallel to $z y$-plane & $120 / 20$ & $120 / 20$ & $120 / 20$ & $120 / 4$ & $300 / 50$ & $300 / 50$ \\
\hline
\end{tabular}

(Model 1 - the anechoic coating layer model in Section 3.1; Model 2 - the anechoic coating layer model in Section 3.2; Model 3 - the local resonance slab model in Section 4.1; Model 4 - the anechoic coating layer model in Section 4.2; Models 5 and 6 - the anechoic coating layer models in Section 4.3).

\subsection{Sound absorption of local resonance slab}

Table 2

Physical parameters of the hard core and silicon rubber.

\begin{tabular}{lllll}
\hline Material & $\rho\left(\mathrm{kg} / \mathrm{m}^{3}\right)$ & $E(\mathrm{~Pa})$ & $v$ & $\mu$ \\
\hline Hard core & 2700 & $7.4 \times 10^{10}$ & 0.33 & 0.0 \\
Silicon rubber & 1300 & $2.0 \times 10^{6}$ & 0.46 & 0.3 \\
\hline
\end{tabular}

The sound absorption coefficient of a local resonance slab backed with orthogonally rib-stiffened plate is also simulated to consolidate the equivalent modulus scheme. For convenience, the sizes of the grating and ribs are set to be the same as above. The scatterer is cylindrical hard core with height of $10 \mathrm{~mm}$ and diameter of $8 \mathrm{~mm}$. The soft silicon rubber has axial thickness of $10 \mathrm{~mm}$ and radial thickness of $2 \mathrm{~mm}$. The physical parameters for all components are constant across frequencies. The host viscoelastic rubber and steel have the same parameters as above and those of the silicon rubber and hard core are listed in Table 2.

Following the same procedures of getting the equivalent moduli of the Alberich anechoic coating, the equivalent moduli and loss factors of the local resonance slab are calculated and shown in Fig. 11(a) and Fig. 11(b), respectively. The calculated 
density of the equivalent homogeneous slab is $1169.1 \mathrm{~kg} / \mathrm{m}^{3}$ and the Poisson's ratio is 0.49. The equivalent moduli are smaller than the original Young's modulus of the host rubber but larger than the equivalent moduli of the Alberich anechoic coating.

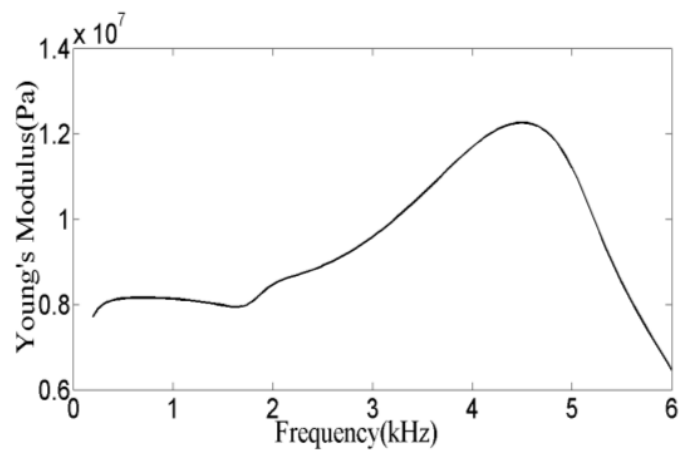

(a)

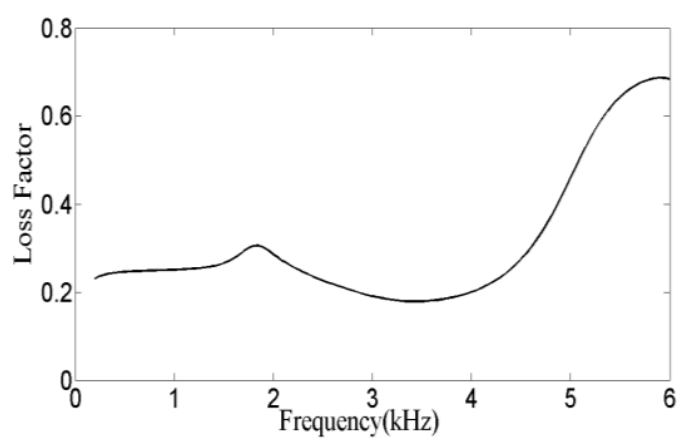

(b)

Fig. 11. Equivalent Young's moduli and loss factors derived from a local resonance slab backed with a steel plate with $13.5 \mathrm{~mm}$ thickness: (a) Young's moduli; (b) loss factors.

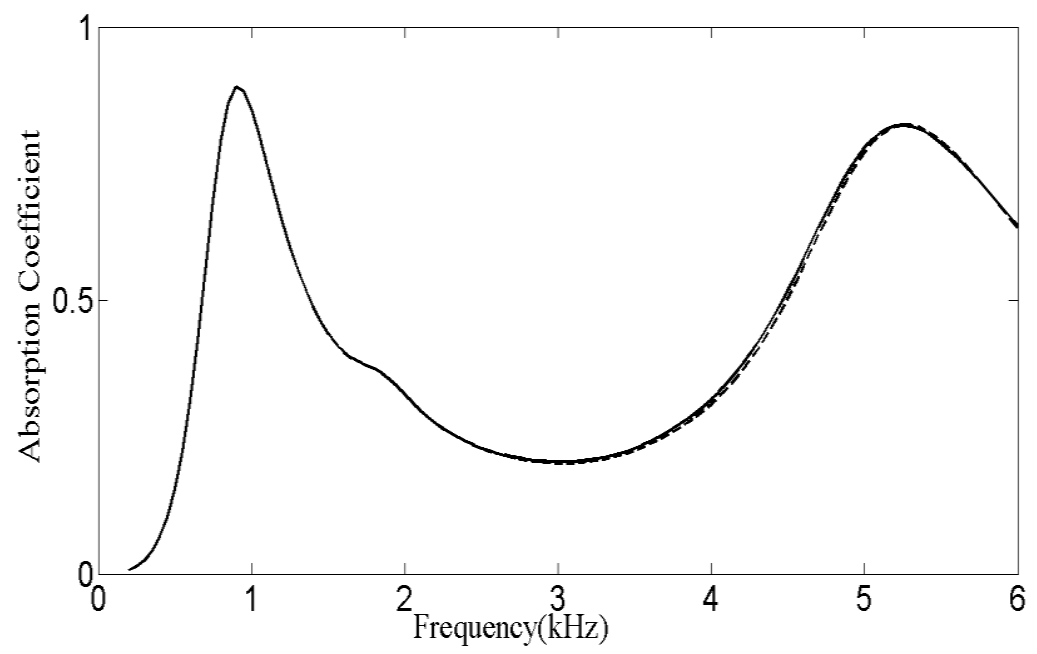

Fig. 12. Absorption coefficients of a local resonance slab and the equivalent homogeneous slab, backed with the same orthogonally rib-stiffened plate. Solid curve: local resonance slab. Dotted curve: equivalent homogeneous slab.

Then, the absorption coefficients of the local resonance slab and the corresponding equivalent homogeneous slab, backed with the same orthogonally rib-stiffened plate are calculated and the results are shown in Fig. 12. The solid curve denotes the result of the original model and the dotted curve represents that of the equivalent model. The two curves are also identical in the whole frequency band, with two peaks located at $900 \mathrm{~Hz}$ and $5400 \mathrm{~Hz}$. 
It is also noted that the original model has about $3.9 \times 10^{4}$ grid nodes, while the equivalent model has about $1 / 10$ of grid nodes, $3.5 \times 10^{3}$.

\subsection{Vibration modal analysis at resonance frequencies}

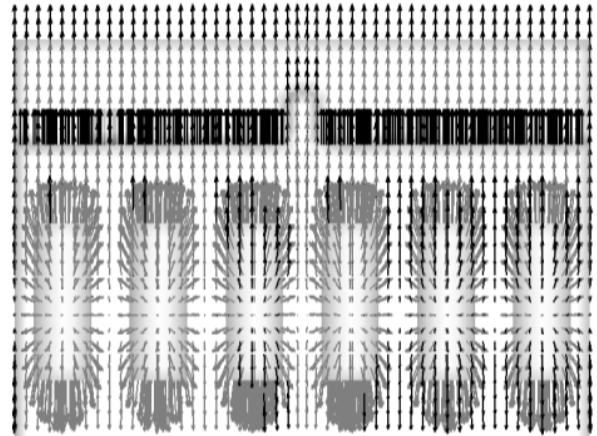

(a)

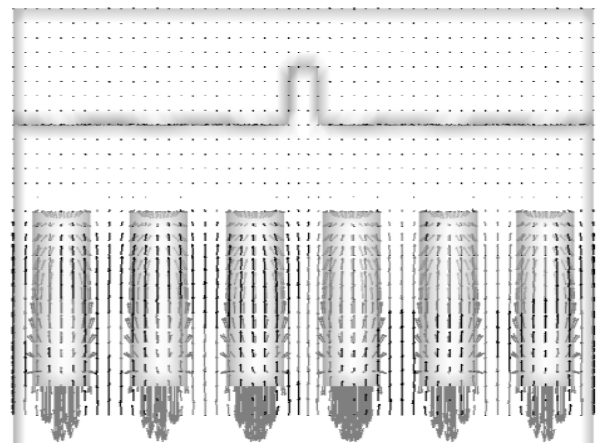

(c)

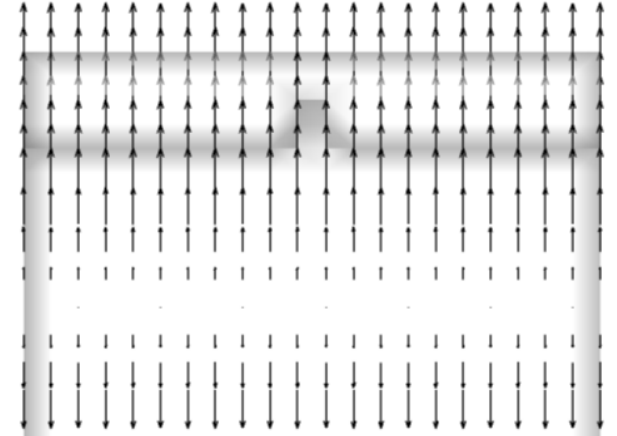

(b)

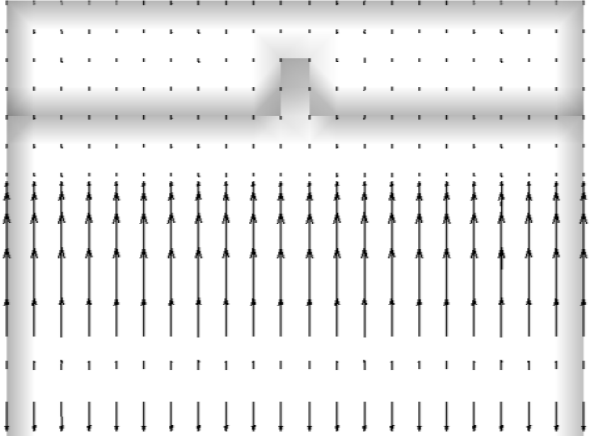

(d)

Fig. 13. Displacement vector graphs in the $z y$-plane of the unit cells of an Alberich anechoic coating and the equivalent homogeneous coating, backed with the same orthogonally rib-stiffened plate: (a) and (c) are the Alberich anechoic coating at $800 \mathrm{~Hz}$ and $4750 \mathrm{~Hz}$, respectively; (b) and (d) are the equivalent homogeneous coating at $800 \mathrm{~Hz}$ and $4750 \mathrm{~Hz}$, respectively.

Further calculation shows that the original and equivalent anechoic coating models backed with orthogonally rib-stiffened plate have the same pattern of modal vibration at resonant frequencies. The displacement vectors in the $z y$-plane at the peak frequencies $(800 \mathrm{~Hz}$ and $4750 \mathrm{~Hz})$ of both models are displayed in Fig. 13. The incident plane wave acts at the bottom of the structure, only the displacement vectors in the solid domain are displayed. Figure 13(a) shows that at the resonant frequency of $800 \mathrm{~Hz}$, the ribs, steel plate and Alberich anechoic coating all vibrate strongly in 
the $z$-axial direction except for a small region across the cavities, where a substantial mode conversion from the longitudinal wave to transverse wave occurs, in a similar manner to the unit cell containing only one cavity in the literature [1]. Figure 13(b) also shows the same pattern of vibration in the equivalent model. At the other resonant frequency of $4750 \mathrm{~Hz}$, the vibration is suppressed by the backing: in the original model in Fig. 13(c), strong vibration only occurs in the coating away from the plate and ribs and the same pattern is also shown in the equivalent model in Fig. 13(d). Based on above analyses, we conclude that, the equivalent anechoic coating plays the same role as the Alberich anechoic coating at peak frequencies in the finite element model. Additionally, the displacement vectors in the $z x$-plane also have the same properties, so they are not displayed.

Figure 13(b) further suggests that the steel plate and ribs work like a mass block. Both components move together and their displacements have the same direction and amplitude. Therefore, the equivalent homogeneous layer can be only stretched in the $z$-axial direction and only vibrate in form of longitudinal wave. In structures with large-size unit cell, however, shear deformation of the equivalent homogeneous layer may be caused by the movement of ribs, so evident mode conversion from longitudinal wave to transverse wave may occur in the homogeneous layer. Such a model is simulated next.

A backed Alberich anechoic coating model with identical geometric parameters as the models in Section 2.2 is constructed. The thickness of the steel plate is assumed to be $4 \mathrm{~mm}$, and ribs $4 \mathrm{~mm}$. The length and height of the rib parallel to the $z y$-plane are $120 \mathrm{~mm}$ and $4 \mathrm{~mm}$, and those of the other rib are $40 \mathrm{~mm}$ and $100 \mathrm{~mm}$. In this way, the unit cell still contains 12 gratings. The physical parameters of the viscoelastic material, steel and water are identical with those described in Section 2.2.

At the first step of the equivalent scheme, the absorption coefficient of an Alberich anechoic coating backed with a $7.7 \mathrm{~mm}$ thick steel plate is calculated (the mean mass of the ribs loaded on one grating of the anechoic coating is added to the original steel plate, so the thickness of the steel plate in the unit cell is increased from $4 \mathrm{~mm}$ to 7.7 $\mathrm{mm}$ ). The calculated absorption coefficient versus frequency is shown in the dotted curve in Fig. 7, in which two peaks at around $1000 \mathrm{~Hz}$ and $4800 \mathrm{~Hz}$ are observed. 
Subsequently, the equivalent Young's moduli and loss factors are calculated and shown in the dotted curves in Fig. 9(a) and Fig. 9(b), respectively. They display a similar trend of variation with the solid curves.

Then, the absorption coefficient of the original model and the corresponding equivalent model are calculated and shown in Fig. 14, in which the result of the original model is displayed in solid line and the result of the equivalent model in dotted line. In addition to two dominant peaks at around $950 \mathrm{~Hz}$ and $4850 \mathrm{~Hz}$ in both the solid and dotted curves, another small peak around $2550 \mathrm{~Hz}$ also appears in the solid curve, and a similar one at around $2700 \mathrm{~Hz}$ in the dotted curve. Below $1950 \mathrm{~Hz}$ the two curves coincide with each other; although their amplitudes are different at frequencies greater than $4300 \mathrm{~Hz}$, the trends of variation are still identical. However, in the frequency range of 1950-4300 Hz, the two curves deviate more from each other, with $250 \mathrm{~Hz}$ shift of the peak frequency. Moreover, compared with Fig. 10, a new low peak appears in this frequency range. Since the only difference in the two models is rib size, this result suggests that different rib design could affect the acoustic performance of Alberich anechoic coating backed by orthogonally rib-stiffened plate.

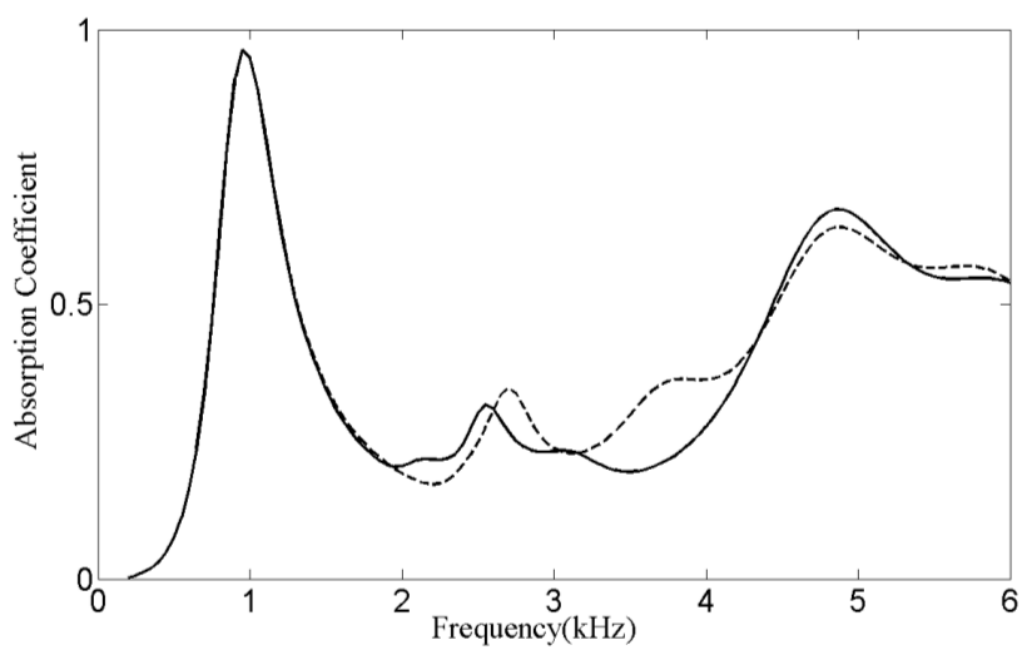

Fig. 14. Absorption coefficients of an Alberich anechoic coating and the equivalent homogeneous coating, backed with the same orthogonally rib-stiffened plate. The rib parallel to the $z x$-plane is much higher than the other one. Solid curve: Alberich anechoic coating. Dotted curve: equivalent homogeneous coating.

In this case, the meshing size of the original model follows the $\lambda / 4$ criterion with respect to flexural wave. The number of grid nodes is $3.2 \times 10^{4}$ and the calculation time 
is 110 hours for 120 frequencies. The equivalent model has $5.2 \times 10^{3}$ and the calculation time is 5 hours. We have noted that the absorption coefficients of the equivalent model are almost the same as those of the original model in the whole frequency band when the meshing size of the equivalent model follows the same criterion of the original mode, suggesting that the calculated absorption coefficients of the equivalent model be sensitive to the grid resolution in the frequency band from $1950 \mathrm{~Hz}$ to $4300 \mathrm{~Hz}$.

The displacement vectors in the $z y$-plane of the original and equivalent models at the three peak frequencies $(950 \mathrm{~Hz}, 2550 \mathrm{~Hz}$ and $4850 \mathrm{~Hz})$ are also shown in Fig. 15. The equivalent model at $2550 \mathrm{~Hz}$ observes the $\lambda / 4$ criterion with respect to flexural wave. Figure 15(a) shows that the whole structure vibrates strongly in the $z$-direction, and the part near the higher rib vibrates more strongly, resulting in non-uniform vibration amplitude in the low rib. The same pattern of vibration is shown in Fig. 15(b). The non-uniform vibration amplitude in the lower rib causes the shear deformation and mode conversion in the equivalent anechoic coating. Compared to Fig. 13(a) in which cavities cause the shear deformation and mode conversion, the wave mode conversion in the Alberich anechoic coating in Fig. 15(a) is more complex because it is caused by both the ribs and cavities.

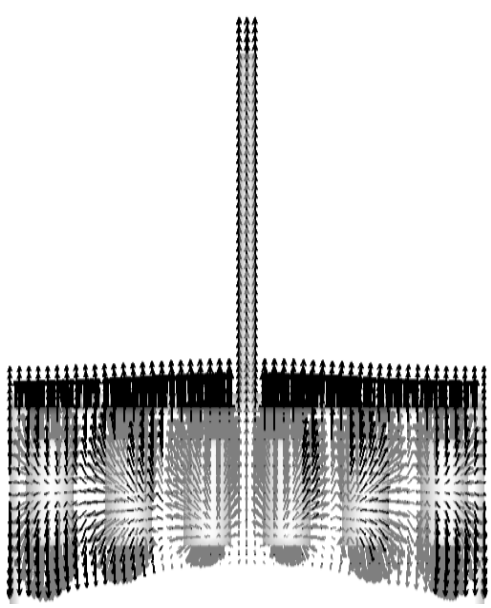

(a)

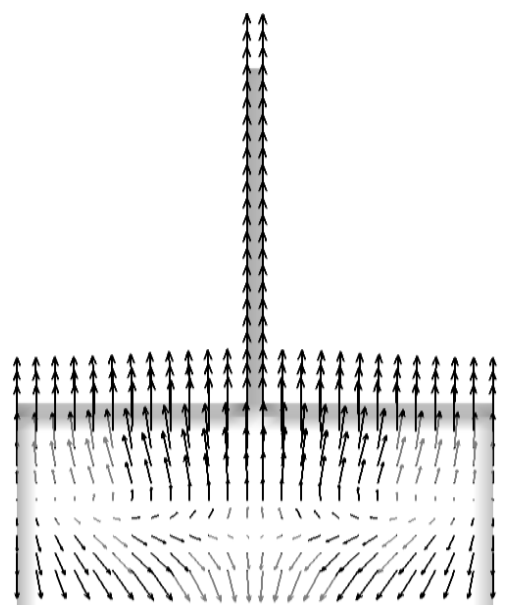

(b) 


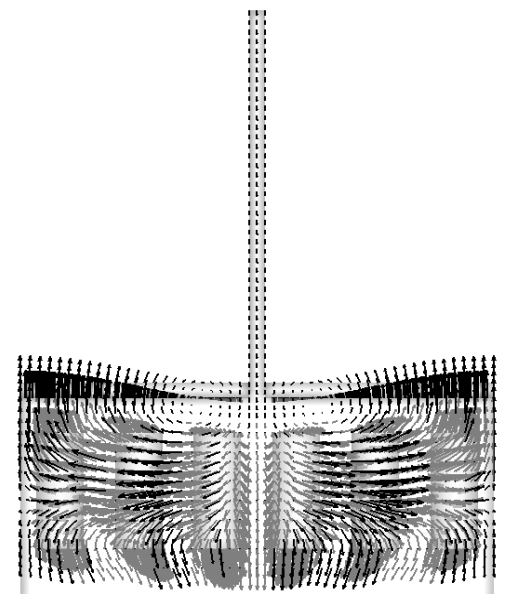

(c)

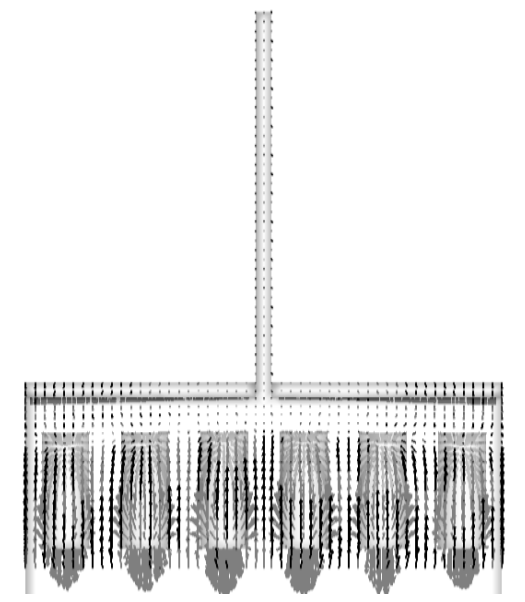

(e)

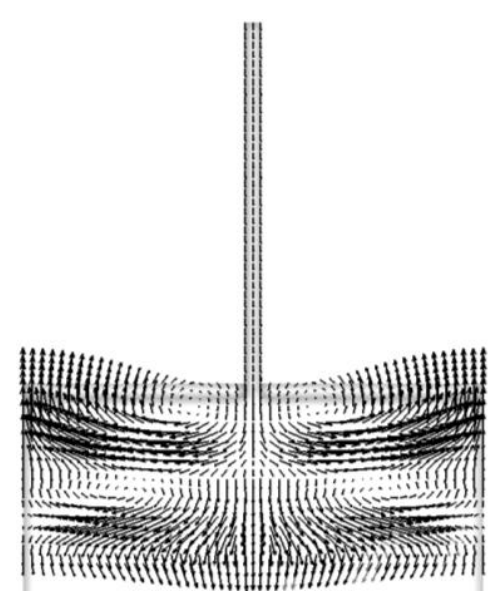

(d)

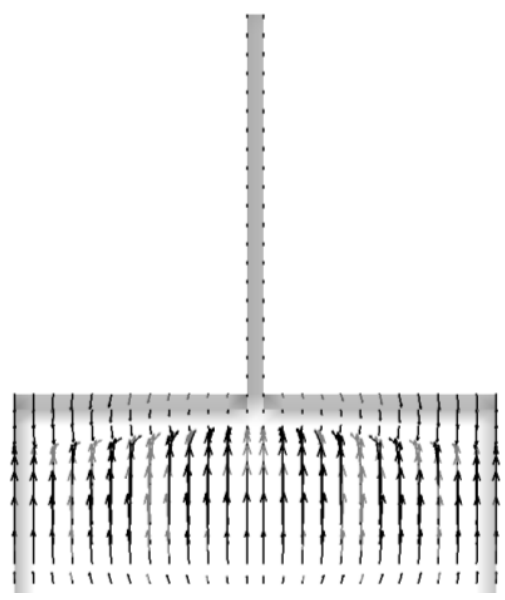

(f)

Fig. 15. Displacement vector graphs in the zy-plane of unit cells of the Alberich anechoic coating and the equivalent homogeneous coating, backed with the same orthogonally rib-stiffened plate. The rib parallel to the $z x$-plane is much higher than the other one. (a), (c), and (e) are the Alberich anechoic coating at $950 \mathrm{~Hz}, 2550 \mathrm{~Hz}$, and $4850 \mathrm{~Hz}$., respectively; (b), (d), and (f) are the equivalent homogeneous coating at $950 \mathrm{~Hz}, 2550 \mathrm{~Hz}$, and $4850 \mathrm{~Hz}$, respectively.

Figure 15(c) and 15(d) suggest that the wave mode conversion not be as vigorous at the peak frequency of $2550 \mathrm{~Hz}$, so the absorption coefficient is not large. The displacement vectors show that the lower rib, steel plate and anechoic coating cause the resonance, but they move in opposite directions, with the vectors on the rib and steel plate pointing to the positive $z$-axis but those on the coating pointing to the negative $z$-axis. In the meanwhile their vibration is suppressed by the higher rib. Therefore, strong vibration only occurs away from the higher rib, causing significant 
shear deformation in the anechoic layer. Although this shear deformation leads to the occurrence of many transverse waves, few of them are transformed from longitudinal vibrations, so wave mode conversion is not so vigorous at this frequency and the absorption coefficient is low.

Figure 15(e) shows strong vibration and wave mode conversion around the cavities at $4850 \mathrm{~Hz}$ in the original model, and Fig. 15(f) also shows that strong vibration only occurs in the equivalent anechoic layer in the equivalent model, suggesting that resonance only occurs in the anechoic coating at $4850 \mathrm{~Hz}$. Both the steel plate and ribs vibrate weakly, resulting in weak shear deformation in the equivalent anechoic coating.

In sum, with the aid of the second equivalent modulus scheme, the sound absorbing properties of an Alberich anechoic coating backed with orthogonally rib-stiffened plate could be predicted with coarsely meshed finite element model. Such model can accurately predict the resonant frequency caused by the whole structure and by the anechoic coating, and also the absorption coefficient around the lower resonant frequency. The predicted absorption coefficient at around the higher resonant frequency may deviate a little from the result of the original finite element model. Moreover, at the resonant frequency when the conditions of ribs cause vigorous shear deformation in the equivalent anechoic coating, refined mesh is required for accurate prediction. Additionally, since a common structure does not have one rib much higher than the other one, shear deformation caused by the ribs would not be so vigorous. Thus, compared with Fig. 14, the absorption coefficient calculated with the equivalent model will accord better with that calculated with the original model.

\subsection{Sound absorption of models with large-size unit cell}

If the unit cell of a model has too large size, it is impossible to directly compute it in a finite element model, and only with the aid of the second equivalent modulus scheme, the absorption performance of an anechoic coating backed with orthogonally rib-stiffened plates can be computed with the given computing capacity. 
Two anechoic coating layer models are constructed, and both have identical geometric parameters except for the thickness of steel plate, one is $10 \mathrm{~mm}$ thick and the other is $6 \mathrm{~mm}$ thick. In the unit cell, the rib parallel to the $z x$-plane is set to be 500 $\mathrm{mm}$ long and $100 \mathrm{~mm}$ high, and the length and height of the other rib are set to be 300 $\mathrm{mm}$ and $50 \mathrm{~mm}$, respectively. The sizes of other parts are identical with the models in Section 2.2, 3.1 and 3.2.

For the thicker model, the unit cell includes 375 gratings, or 375 cavities, and their directly meshed finite element models would have more than $1.0 \times 10^{6}$ grid nodes if the $\lambda / 4$ criterion is observed with respect to the flexural wave, making the numerical operation of fluid-structure interaction extremely difficult. In contrast, after the equivalent modulus method is applied, coarse meshes with $3.5 \times 10^{4}$ grid nodes are sufficient to solve the same problem.

In implementing the equivalent modulus scheme, the absorption coefficients of the Alberich anechoic coating backed with steel plates with thicknesses of $12.6 \mathrm{~mm}$ and $8.6 \mathrm{~mm}$ are firstly calculated to obtain the equivalent physical parameters. The results are very close to those shown in Fig. 7, and the calculated equivalent Young's moduli and loss factors for both models are also very close to the dotted curves drawn in Fig. 9(a) and Fig. 9(b), respectively, so they are not displayed again.

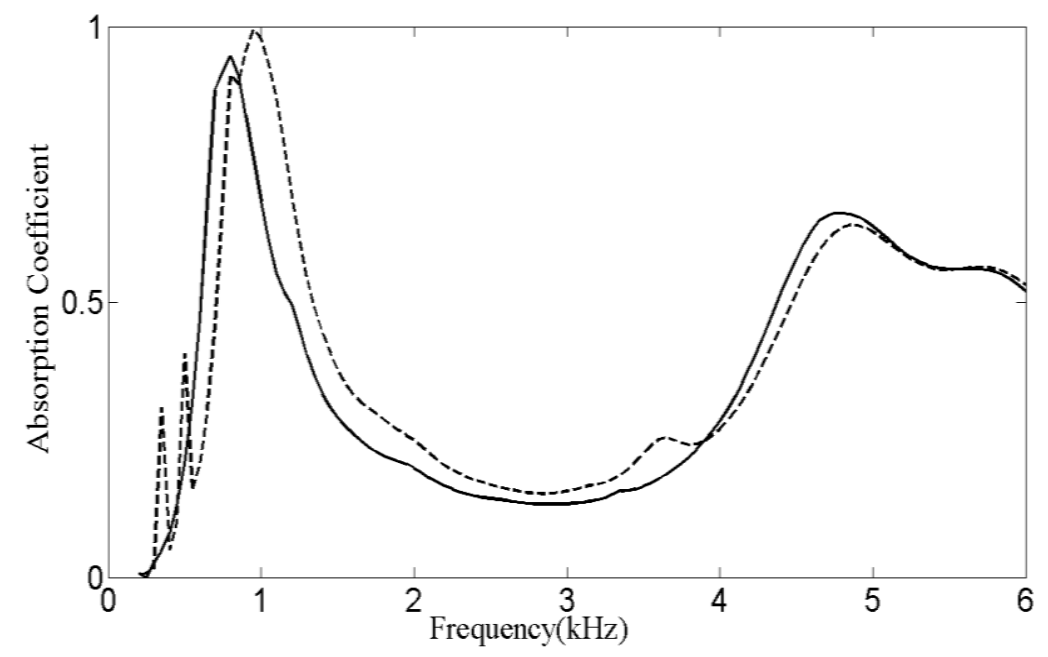

Fig. 16. Absorption coefficients of the anechoic coating backed with orthogonally rib-stiffened plates with large-size unit cells. Solid line: plate with $10 \mathrm{~mm}$ thickness. Dotted line: plate with 6 mm thickness. 
The calculated absorption coefficient versus frequency for both models are shown in Fig. 16, in which the solid and dotted curves denote the results of the model with $10 \mathrm{~mm}$ and $6 \mathrm{~mm}$ thick steel plate, respectively. Two absorption peaks at around 800 $\mathrm{Hz}$ and $4800 \mathrm{~Hz}$ appear in the solid curve for the model with thicker steel plate; For the model with thinner steel plate, besides two main peaks at around $950 \mathrm{~Hz}$ and 4850 $\mathrm{Hz}$, three other lower peaks at around $350 \mathrm{~Hz}, 500 \mathrm{~Hz}$ and $3650 \mathrm{~Hz}$, and another higher peak around $800 \mathrm{~Hz}$ appear in the dotted curve. It is noted that, for the structure with large-size unit cell, the reflection coefficient $R$ is not only derived from one plane wave, but also made up of the reflection coefficients of other higher order waves. The theoretical details can be found in references [4], [6], [7] and [11].

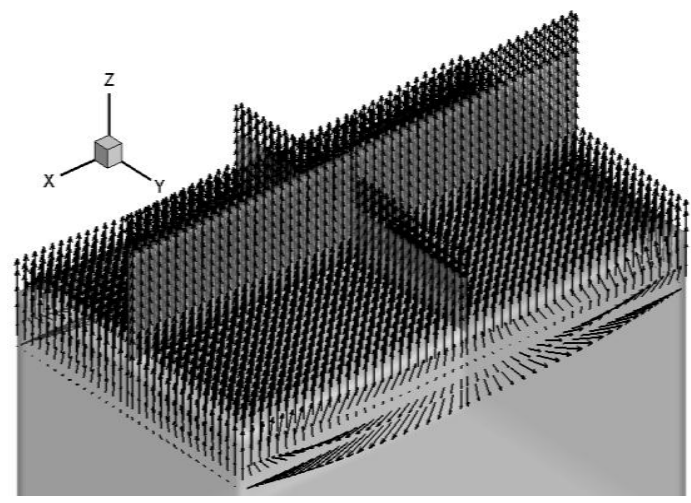

(a)

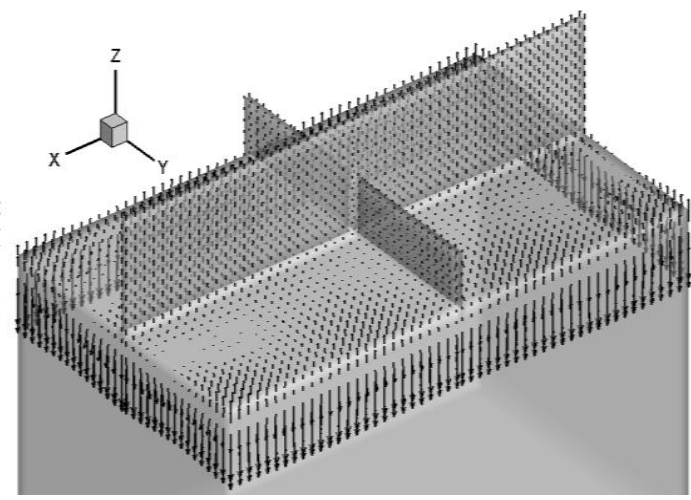

(b)

Fig. 17. 3-D displacement vector graphs of the unit cell of an equivalent anechoic coating backed with a large-size orthogonally rib-stiffened plate, at different resonant frequencies. The plate is 10 mm thick: (a) at the resonant frequency of $800 \mathrm{~Hz}$. (b) at the resonant frequency of $4800 \mathrm{~Hz}$.

The displacement vectors of the structure at major peak frequencies are also displayed for the two models. The model sizes in the $x$-axis and $y$-axis are so large that shear deformation occurs in both the $z x$ and $z y$-cross sections, so 3-D graphs are presented for better depiction.

The displacement vectors of the model with $10 \mathrm{~mm}$ thick steel plate at $800 \mathrm{~Hz}$ are shown in Fig. 17(a), and those at $4800 \mathrm{~Hz}$ are shown in Fig. 17(b). The displacement of the fluid domain below the structure is set zero in the figure. The whole structure 
resonates at $800 \mathrm{~Hz}$ but only the equivalent anechoic layer resonates at $4800 \mathrm{~Hz}$. Some wave mode conversion caused by the ribs occurs in the equivalent anechoic layer at the two resonant frequencies.

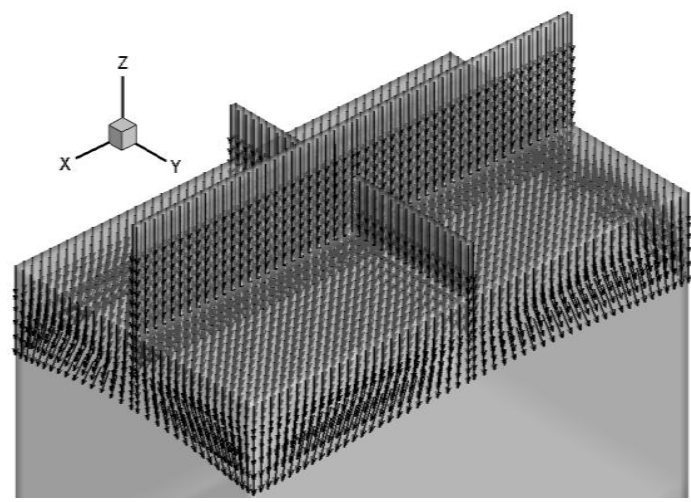

(a)

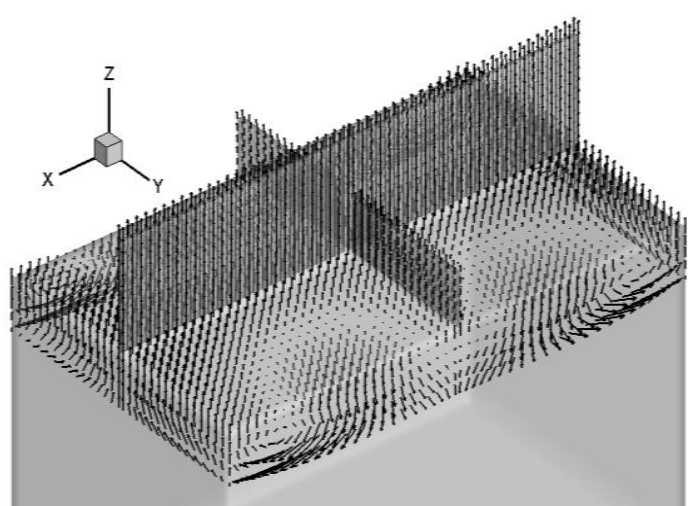

(c)

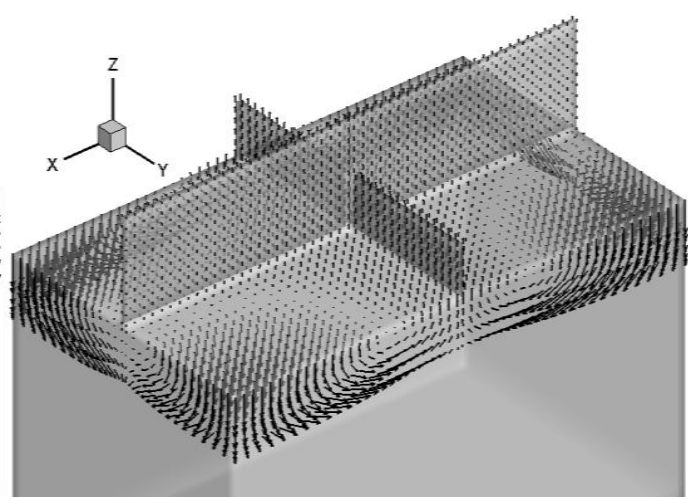

(b)

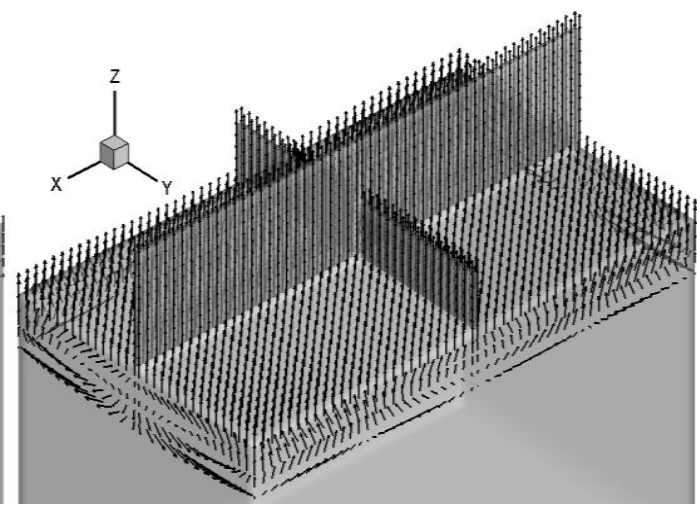

(d)

Fig. 18. 3-D displacement vector graphs of the unit cell of an equivalent anechoic coating backed with a large-size orthogonally rib-stiffened plate, at different resonant frequencies. The plate is 6 mm thick: (a) at the resonant frequency of $350 \mathrm{~Hz}$; (b) at the resonant frequency of $500 \mathrm{~Hz}$. (c) at the resonant frequency of $800 \mathrm{~Hz}$. (d) at the resonant frequency of $950 \mathrm{~Hz}$.

Figures 18(a), 18(b), 18(c), and 18(d) show the 3-D displacement vectors of the model with $6 \mathrm{~mm}$ thick steel plate at resonant frequencies of $350 \mathrm{~Hz}, 500 \mathrm{~Hz}, 800 \mathrm{~Hz}$, and $950 \mathrm{~Hz}$, respectively. At $350 \mathrm{~Hz}$, the whole structure resonates; however, the wave length is much larger than the size of the model, so most of the wave propagates back to the water, resulting in a lower resonant absorption peak. At $500 \mathrm{~Hz}$, the lower 
rib, steel plate and anechoic coating resonate, but they move in opposite directions, with the vectors on the rib pointing to the positive $z$-axis, while those on the plate and coating pointing to the negative $z$-axis. Hence, the vibration of the lower rib partially counteracts that of the steel plate and anechoic coating, so the absorption peak is low. At $800 \mathrm{~Hz}$, the higher rib, steel plate and anechoic coating resonate, and vigorous wave mode conversion occurs around the cavities in the corresponding original model, resulting large absorption coefficient. At $950 \mathrm{~Hz}$, the whole structure resonates and some shear deformation caused by the ribs occurs in the equivalent anechoic layer. At $3650 \mathrm{~Hz}$, a complex resonance occurs at this frequency, in which the vibration of the higher rib partially counteracts that of the lower rib, resulting in a small absorption coefficient. At $4850 \mathrm{~Hz}$, the displacement vectors are very similar to those in Fig. 17(b) (The displacement vectors at $3650 \mathrm{~Hz}$ and $4850 \mathrm{~Hz}$ are not shown).

The results of the two models suggest that for the Alberich anechoic coating backed with orthogonally rib-stiffened plates, some new resonant modes may appear if the steel plate is thin. These new resonance may be caused by the vibration of higher rib, steel plate and anechoic coating layer, which would significantly boost sound absorption. In this study, the absorption coefficient is 0.91 near the first main resonant frequency, and less than 0.5 around the other new resonant frequencies. It can be also inferred that with the increase of unit cell size, more resonances might occur.

\section{General discussions on the first absorption peak}

Comparison of the absorption coefficients of the Alberich anechoic coating with air backing (Fig. 4) and the Alberich anechoic coating with orthogonally rib-stiffened steel plate (Fig. 16) shows that the first main absorption peak shifts to a substantially lower frequency range because of the added mass of steel plate and ribs. The resonance at the first main peak frequency is similar to a spring-mass oscillator, in which the backing works as the mass block, hence the heavier backing brings lower peak frequency in Fig. 16. This behavior is identical with that mentioned in previous literature [1]. 
The first main peak frequency is also affected by the shear deformation caused by ribs, but less significantly. For example, for two structures with identical mean backing mass loaded on one grating of the anechoic coating, the first main peak of the dotted curve is $1000 \mathrm{~Hz}$ in Fig. 7, while that in Fig. 14 is $950 \mathrm{~Hz}$. The latter model has vigorous shear deformation, which causes the fall of the first main peak frequency.

Moreover, heavier backing can decrease the absorption coefficient at the first main peak. The absorption coefficient is 0.99 at $2150 \mathrm{~Hz}$ for the Alberich anechoic coating with air backing in Fig. 4, 0.96 at $950 \mathrm{~Hz}$ for the Alberich anechoic coating backed with orthogonally rib-stiffened steel plate in Fig. 14, and 0.90 and 0.94 at $800 \mathrm{~Hz}$ for the Alberich anechoic coating with heavier backings in Fig. 10 and 16, respectively. The orthogonally rib-stiffened plate not only increases the mass of the backing, which shifts the absorption peak to a lower frequency, but also suppresses the vibration of the anechoic coating, resulting in decreased absorption coefficient at the peak frequency.

On the other hand, the shear deformation caused by ribs can slightly increase the absorption coefficient. For the Alberich anechoic coating with heavier backings in Fig. 10 and 16 , the absorption coefficients are 0.90 and 0.94 , respectively at the same peak frequency $(800 \mathrm{~Hz})$. The displacement vectors of the former model shown in Fig. 13(b) have no apparent appearance of shear deformation, but shear deformation is observed in the displacement vectors of the latter model shown in Fig. 17(a). Moreover, more vigorous shear deformation appears in Fig. 18(d), occupying both the $z x$ and $z y$-planes; therefore, higher absorption coefficient of 0.99 is found at the corresponding peak frequency $(950 \mathrm{~Hz})$.

\section{Conclusions}

Two equivalent modulus schemes are proposed for the homogenization of the finite element model of anechoic coating backed with orthogonally rib-stiffened plate, and the sound absorption coefficient of the same structure is derived with the two schemes. The simulated absorption coefficients suggest that compared with the first scheme and 
the method in reference [11], the second scheme be more effective in a wider low frequency band, the simulated result agrees with the result simulated with the original finite element model at the two main absorption peaks in the low frequency range. The application of the method has been taken a good advantage to avoid the large number of grid nodes of a large-size unit cell. With this method, the number of grid nodes of the finite element meshes is also greatly reduced $\left(3.8 \times 10^{4}\right.$ vs $\left.3.5 \times 10^{3}\right)$.

The second equivalent modulus scheme is not only suitable for the model with voided cavities, but also for the slab model with local resonance. Moreover, at the resonant frequency when the conditions of ribs cause vigorous shear deformation in the equivalent anechoic coating, refined mesh is required for accurate prediction.

The simulation results also give some new hints about the sound absorption coefficient of anechoic coating backed with orthogonally rib-stiffened plate:

(1) Such structure with large-size unit cells has two main absorption peaks, and some lower absorption peaks (most with amplitudes smaller than 0.5) occur at new resonant frequencies when the steel plate becomes thinner. These new resonance may be caused by the vibration of higher rib, steel plate and anechoic coating layer,

(2) Comparison of the first main absorption peaks of the anechoic coating with different backings shows that increased mass of the backing shifts the absorption peak to a low frequency, but it also suppresses the vibration of the anechoic coating so the absorption coefficient is decreased at the first peak frequency. For the structures with large-size unit cells, the shear deformation caused by the ribs can mildly increase the absorption coefficient at the first peak frequency.

\section{Acknowledgements}

This work is supported by the National Science Foundation of China (11374326), State Key Development Program for Basic Research of China (973 Program: 2012CB720204), China Postdoctoral Science Foundation (2014M550853), and National Science Foundation of China (11404368). 


\section{References}

[1] H. Meng, J.H. Wen, H.G. Zhao, L.M. Lv, X.S. Wen, Analysis of absorption performances of anechoic layers with steel plate backing, Journal of the Acoustical Society of America 132 (2012) $69-75$.

[2] T.C. Ma, R.A. Scott, W.H. Yang, Harmonic wave propagation in an infinite elastic medium with a periodic array of cylindrical pores, Journal of Sound and Vibration 71 (1980) 473-482.

[3] A.C. Hennion, R. Bossut, J.N. Decarpigny, C. Audoly, Analysis of the scattering of a plane acoustic wave by a periodic elastic structure using the finite element method: Application to compliant tube gratings, Journal of the Acoustical Society of America 87 (1990) 1861-1870.

[4] A.C. Hennion, J.N. Decarpigny, Analysis of the scattering of a plane acoustic wave by a doubly periodic structure using the finite element method: Application to Alberich anechoic coatings, Journal of the Acoustical Society of America 90 (1991) 3356-3367.

[5] V. Easwaran, M.L. Munjal, Analysis of reflection characteristics of a normal incidence plane wave on resonant sound absorbers: A finite element approach, Journal of the Acoustical Society of America 93(1993) 1308-1318.

[6] J.H. Wen, H.G. Zhao, L.M. Lv, B. Yuan, G. Wang, X.S. Wen, Effects of locally resonant modes on underwater sound absorption in viscoelastic materials, Journal of the Acoustical Society of America 130 (2011) 1201-1208.

[7] H. Meng, J.H. Wen, H.G. Zhao, L.M. Lv, X.S. Wen, Optimization of locally resonant acoustic metamaterials on underwater sound absorption characteristics, Journal of Sound and Vibration 331 (2012) 4406-4416.

[8] F.X. Xin, T.J. Lu, Analytical modeling of fluid loaded orthogonally rib-stiffened sandwich structures: Sound transmission, Journal of the Mechanics and Physics of Solids 58 (2010) 1374-1396.

[9] A.J. Hull, J.M. Maguire, Elastic response of an orthogonally reinforced plate, Journal of Sound and Vibration 333 (2014) 2327-2346.

[10] M.C. Remillieux, R.A. Burdisso, Vibro-acoustic response of an infinite, rib-stiffened, thick-plate assembly using finite-element analysis, Journal of the Acoustical Society of America 132 (2012) EL36-EL42.

[11] X.Y. Fu, Z.K. Jin, Y. Yin, B.L. Liu, Sound absorption of a rib-stiffened plate covered by anechoic coatings, Journal of the Acoustical Society of America 137 (2015) 1551-1556.

[12] M.-F. Poujol-Pfefer, Application of an homogenization model to the acoustical propagation in inhomogeneous media, Journal of Sound and Vibration 184 (1995) 665-679.

[13] G.C. Gaunaurd, W. Wertman, Comparison of effective medium theories for inhomogeneous continua, Journal of the Acoustical Society of America 85 (1989) 541-554.

[14] D. Jiang, D.H. Zhang, Q.G. Fei, S.Q. Wu, An approach on identification of equivalent properties of honeycomb core using experimental modal data, Finite Elements in Analysis and Design 90 (2014) 84-92. 
[15] D.H. Chen, L. Yang, Analysis of equivalent modulus of asymmetrical honeycomb, Composite Structures 90 (2011) 767-773.

[16] K.D. Belder, R. Pintelon, C. Demol, P. Roose, Estimation of the equivalent complex modulus of laminated glass beams and its application to sound transmission loss prediction, Mechanical System and Signal Processing 24 (2010) 809-822.

[17] J. Allard, N. Atalla, Propagation of sound in porous media: modelling sound absorbing materials, 2th ed, United Kingdom: John Wiley \& Sons 2009.

[18] J.C. Walter, G.T. Barkema, An introduction to Monte Carlo method, Physica A 418 (2015) 78-87. 